\title{
Application of a "United Scoring Method" to Patients with Traumatic Brain Injury in the Critically Surgical State
}

\author{
Kaimin Wang1,2, Hao Li1,2, Zhiqiang $\mathrm{Li}^{2}$, Ning Zhang², Zhe Quan² \\ ${ }^{1}$ Southern Medical University, Guangzhou, China \\ ${ }^{2}$ Department of Neurosurgery, Fengxian District Central Hospital (Fengxian Affiliated Hospital of Southern \\ Medical University), Shanghai, China \\ Email:wkm20120910@163.com, 438322648@qq.com, "Izq_99968@163.com, ginoning@126.com, \\ superquanzhe@163.com
}

Received 19 May 2016; accepted 12 June 2016; published 16 June 2016

Copyright (C) 2016 by authors and OALib.

This work is licensed under the Creative Commons Attribution International License (CC BY). http://creativecommons.org/licenses/by/4.0/

(c) (i) Open Access

\begin{abstract}
Introduction: To investigate a more reasonable treatment strategy for traumatic brain injury in the critical surgery state and increase the clinical therapeutic effects. Methods: Fifty-nine patients with traumatic brain injury in the critical operation state were randomly divided into an experimental group $(n=31)$ and a control group $(n=28)$. Patient's prognosis was compared between these two groups. Results: According to the Glasgow Outcome Scale scoring, 23 patients presented with low disability, 7 with moderate disability, 1 with severe disability, and no patients were in the persistent vegetative state or died in the experimental group; 14 patients presented with low disability, 8 with moderate disability, 3 with severe disability, 2 were in the vegetative state, and 1 died in the control group. Patient's prognosis in the experimental group was significantly better than that in the control group $(P<0.05)$. Conclusion: The combined scoring system based on Glasgow Outcome Scale, intracranial pressure value and CT findings is of significance in the management of patients with traumatic brain injury in the critical surgery state.
\end{abstract}

\section{Keywords}

Traumatic Brain Injury, Indication for Surgery, Critical Surgery State

Subject Areas: Neurology

${ }^{*}$ Corresponding author.

How to cite this paper: Wang, K.M., Li, H., Li, Z.Q., Zhang, N. and Quan, Z. (2016) Application of a "United Scoring Method" to Patients with Traumatic Brain Injury in the Critically Surgical State. Open Access Library Journal, 3: e2713.

http://dx.doi.org/10.4236/oalib.1102713 


\section{Introduction}

Traumatic brain injury is a complex injury and its therapeutic effects are affected by a series of factors including patient's age, gender, severity of disease (Glasgow Coma Scale (GCS) score, presence of brain hernia, cisterna ambiens status, combined injury, and multiple injury), basic diseases and timely treatment [1] [2]. A better understanding of indications for surgery is extremely important for patients with traumatic brain injury. However, in the clinical practice, some patients have traumatic brain injury in the critical surgery state, which brings difficulties in decision-making for physicians. In this study, the patients were scored according to a combined scoring system, i.e., GOS score, intracranial pressure value, and CT findings regarding brain injury, which is commonly used in the field of neurosurgery. Encouraging outcomes have been acquired and are reported as follows.

\section{Materials and Methods}

\subsection{Clinical Materials}

Fifty-nine patients with traumatic brain injury in the critical surgery state who received treatment between May 2012 and May 2014 were included in the experimental group. These patients, consisting of 24 males and 7 females, were aged 20 - $65(43.2 \pm 7.3)$ years. Causes of traumatic brain injury in these patients included traffic accidents $(n=26)$, high falls $(n=4)$ and collision $(n=1)$. Intracranial injury types included acute subdural hematoma $(n=18)$, acute subdural hematoma accompanied by intracerebral hematoma $(n=6)$, brain contusion accompanied by hematoma $(n=6)$, and unilateral subdural hematoma accompanied by contralateral epidural hematoma $(n=1)$. Twenty-eight patients, 22 males and 6 females, aged $20-78$ (42.7 \pm 7.6$)$ years, were included in the control group. Causes of traumatic brain injury in these control patients included traffic accidents $(n=24)$, high falls $(n=3)$ and collision $(n=1)$. Intracranial injury types included acute subdural hematoma $(n=$ 17), acute subdural hematoma accompanied by intracerebral hematoma $(n=5)$, brain contusion accompanied by hematoma $(n=5)$, and unilateral subdural hematoma accompanied by contralateral epidural hematoma $(n=1)$. There were no significant differences in gender, age, injury causes and disease severity between experimental and control groups $(P>0.05)$.

\subsection{Inclusion Criteria}

According to the traumatic brain injury indications for surgery designated by Wu et al. [3], patients with traumatic brain injury in the critical surgery state were included.

\subsection{Exclusion Criteria}

Patients not correspond to inclusion criteria; pregnant and lactating women; accompanied by severe internal diseases; uncooperative patients or those who withdraw from treatment without authorization; those cannot provide complete data.

\subsection{Methods}

The conservative and surgical treatments were relatively consistent between the experimental and control groups.

\subsection{Experimental Group}

On admission, experimental group patient's GCS score, intracranial pressure value and CT findings were recorded (time unit 2 hours; hematoma volume measured in $\mathrm{mL}$; intracranial pressure value measured in mmHg). Patients were scored according to the records.

\subsection{Scoring Rules Are as Follows}

Clinical signs: a score of 1 was given when GCS score was decreased by every 1 point compared to the last GCS evaluation according to a comprehensive evaluation of GCS score, intracranial pressure value and CT findings.

Intracranial pressure value: Following the descriptions by Zhou et al. and the consensus from experts [4] [5]: a score of 1 was given when intracranial pressure value was $20 \mathrm{mmHg}$, a score of 2 for $30 \mathrm{mmHg}$ intracranial 
pressure value, a score of 3 for $40 \mathrm{mmHg}$ intracranial pressure and the like.

CT findings: a score of 1 was given when hematoma volume (calculated by Tada formula [6]) was $20 \mathrm{~mL}$, a score of 2 for $30 \mathrm{~mL}$ hematoma volume, a score of 3 for $40 \mathrm{~mL}$ hematoma volume, and the like. If hematoma occurred at the pars temporalis, 1 more point was added; a score of 2 was given when posterior fossa hematoma reached $5 \mathrm{~mL}$, a score of 3 for $10 \mathrm{~mL}$ posterior fossa hematoma, a score of 4 for $15 \mathrm{~mL}$ posterior fossa hematoma, and the like.

A score of 1 was given when the cerebral ventricle was slightly compressed ( $1 / 3$ cerebral ventricle was occluded), a score of 2 for moderately compressed cerebral ventricle (2/3 cerebral ventricle was occluded), a score of 3 for severely compressed cerebral ventricle (the whole cerebral ventricle was occluded); a score of 1 was given when median line was $0.5 \mathrm{~cm}$ shifted, a score of 2 for $1.0 \mathrm{~cm}$ shifting and a score of 3 for $1.5 \mathrm{~cm}$ shifting.

A score of 1 was given when the cistern ambiens was slightly occluded [7] (width $1-2 \mathrm{~mm}$ ), a score 2 for moderate occlusion (width $<1 \mathrm{~mm}$ ) and a score of 3 for severe occlusion (cistern ambiens disappear).

Scoring threshold for surgery: Total scores were the sum of GCS score and other scores given according to intracranial pressure value and CT findings. Conservative treatment was recommended for total scores of $<5$ points, surgery was recommended for total scores being $5-7$ points, and surgery was highly recommended for total scores of $>7$ points.

\subsection{Control Group}

According to the description by Wu et al. [3], control group patients were treated as per traumatic brain injury. Surgery was performed when disease condition was aggravated and indications for surgery were identified.

\subsection{Follow Up}

Six months later, all survivors were followed up and received neurological system examination. They were graded according to GOS scores, and their prognosis was analyzed and evaluated.

\subsection{Evaluation Criteria}

All included patients were assessed according to GOS rating: grade I, dead; II, vegetative state (long-term unresponsiveness, decerebrate rigidity); III, severely disabled (with permanent need for help with daily living); IV, moderately disabled (the patient is independent but disabled); V, good recovery (the patient has resumed most normal activities but may have minor residual problems).

\subsection{Statistical Analysis}

SPSS20.0 software was used for statistical analysis. The Wilcoxon rank-sum test was used for comparison of numeration data between experimental and control groups. A level of $P \leq 0.05$ was considered statistically significant.

\section{Results}

All survivors were followed up after 6 months. According to GOS grade, patient's prognosis is shown in Table 1. The results of the Wilcoxon rank-sum test are displayed in Table $2(\mathrm{Z}=-2.184, P=0.029<0.05)$.

\section{Discussion}

Traumatic Brain Injury (TBI) is a leading cause of mortality and disability in the field of neurosurgery. With the wide application of CT technology and intracranial pressure monitoring and the development of life support technology, the mortality of severe traumatic brain injury is gradually decreased. After popularization of brain injury treatment guidelines issued by every country and the constant normalized training to physicians at all levels, in particular after intensive use of intracranial pressure monitoring, the mortality of severe traumatic brain injury is decreased from approximately $40 \%$ in the 1890 s to about $20 \%$ at present [8]. Increasing evidence has demonstrated that use of intracranial pressure monitoring in patients with traumatic brain injury obviously improves patient's prognosis because of increased attention to patients and increased presentation of patient's condition as well as the therapeutic effect of ventricular probe. Farahvar et al. [9] analyzed the data of 1446 patients 
Table 1. Comparison of patient’s prognosis between experimental and control groups.

\begin{tabular}{cccc}
\hline Prognosis & Experimental group & Control group & Total \\
\hline Grade I (dead) & 0 & 1 & 1 \\
Grade II (vegetative state) & 0 & 2 & 2 \\
Grade III (severely disabled) & 1 & 3 & 4 \\
Grade IV (moderately disabled) & 7 & 8 & 15 \\
Grade V (good recovery) & 23 & 14 & 37 \\
Total & 31 & 28 & 59 \\
\hline
\end{tabular}

\begin{tabular}{cccc}
\hline \multicolumn{4}{l}{ Table 2. The Wilcoxon rank-sum test results of patient’s prognosis. } \\
\hline Group & Number of patients & Mean rank & Rank \\
\hline Experimental & 31 & 33.98 & 1053.50 \\
Control & 28 & 25.59 & 716.50 \\
Total & 59 & & \\
\hline
\end{tabular}

$\mathrm{Z}=-2.184, P=0.029$ (bilateral). Patient's prognosis in the experimental group was significantly better than that in the control group (33.98 > 25.59).

with severe traumatic brain injury and they found that after 2 weeks, the death rate of 1202 patients who received intracranial pressure monitoring was significantly lower than that of 244 patients without receiving intracranial pressure monitoring. Alali et al. [10] analyzed the clinical data of 10,628 patients with severe traumatic brain injury from 155 hospitals in the USA and Canada. Results showed that the death rate of hospitalized patients receiving intracranial pressure monitoring was lower than that of non-hospitalized patients. These results suggest the importance of intracranial pressure monitoring.

Surgical treatment is an important mean to save the life of patients with severe traumatic brain injury. In China, surgical indications for traumatic brain injury are commonly used according to the teaching books written by Wu et al. [3] and Chen et al. [11]. But when patient's disease condition, intracranial pressure value and CT findings or one or two items of them are in the critical surgery state, there is a divergence regarding whether surgical treatment is used between different physicians. Neurological function examination is often limited by poor sensitivity and other factors. The Glasgow Coma Scale (GCS) can not accurately reflect the practical situation of every patient with severe traumatic brain injury. CT can help display the degree and scope of traumatic brain injury [12], providing guidance for evaluation of traumatic brain injury, but it also displays different information from practical situation.

Therefore, we graded the patients with traumatic brain injury according to their clinical signs (GCS scores), intracranial pressure value and CT findings, providing quantitative indices and evidence for selecting solutions by clinical physicians and showing clinical application prospect. More studies are required to further confirm its clinical value to be popularized, providing guidance for managing patients with incurable traumatic brain injury, helping clinical physicians to better take the opportunity for surgery, which can reduce unnecessary costs, physician's invalid labor and the risks for death.

\section{Conclusion}

The "united scoring method" developed based on Glasgow Outcome Scale, intracranial pressure value and CT imaging findings is a simple and quantitative method. It can be used for patients with traumatic brain injury in the critically surgical state and some therapeutic effects have been acquired. It overcomes the limitation that whether surgery is performed only according to one reference factor. This "united scoring method" possesses application prospect and it holds the promise that it can help clinical physicians to seize the opportunity for performing surgery as early as possible in the patients who meet the surgical indications to increase therapeutic efficacy. In addition, it can also help determine whether conservative treatment should be performed; thus, unne- 
cessary pains and economic loss can be reduced. Therefore, this "united scoring method" is of important significance in the diagnosis and treatment of traumatic brain injury patients.

\section{Key Messages}

- The data used in the "united scoring method" are easy to be collected and are the common indices of physical examination.

- The "united scoring method" emphasizes to take the three important factors that are commonly used in the clinic to judge the condition of disease into consideration.

- In the preliminary experiment, the detailed score information is achieved, which can provide quantitative criteria and facilitate grading.

- The "united scoring method" overcomes the limitation that whether surgery is performed according to one reference factor. For example, intracranial pressure influences the decision-making of secondary surgery.

- The "united scoring method" is a novel design and needs to be further studied in the practice.

- The "united scoring method" is suitable for the common problems encountered in the clinical practice.

\section{Competing Interests}

The authors declare that they have no competing interests.

\section{Authors' Contributions}

Zhi-Qiang Li conceived, designed the experiments, and wrote the paper. Hao Li and Bo Ma performed the experiments. Ning Zhang and Zhe Quan analyzed the data and provided reagents/materials/analysis tools. Zhi-Qiang $\mathrm{Li}$ was responsible for this article. All authors read and approved the final manuscript.

\section{References}

[1] Wang, B.H., Hui, G.Z. and Pei, Y.E. (2006) Analysis on Factors Influencing the Therapeutic Effects of Acute Traumatic Extradural Hematoma (a Report of 484 Cases). Chinese Journal of Clinical Neurosurgery, 11, 341-343.

[2] Li, Z.Q., Shen, D.Q., Quan, Z., Ming, C. and Ying, L. (2009) Analysis on Prognosis-Related Factors in Patients with Severe Traumatic Brain Injury. Chinese Journal of Primary Medicine and Pharmacy, 16, 1361-1362.

[3] Wu, Z.D. and Wu, Z.H. (2010) Surgery Science. 7th Edition, People’s Medical Publishing House, Beijing.

[4] Kiehna, E.N., Huffmyer, J.L., Thiele, R.H., Scalzo, D.C. and Nemergut, E.C. (2013) Use of the Intrathoracic Pressure Regulator to Lower Intracranial Pressure in Patients with Altered Intracranial Elastance: A Pilot Study. Journal of Neurosurgery, 119, 756-759. http://dx.doi.org/10.3171/2013.4.JNS122489

[5] (2011) Chinese Congress of Neurological Surgeons, Chinese Committee of Neurological Injury Experts: Chinese Expert Consensus on Intracranial Pressure Monitoring in Craniocerebral Trauma. Chinese Journal of Neurosurgery, 27, 1073-1074.

[6] Yan, Y.K., Liu, H.J., Li, J.Y., Cao, H.Z., Yan, C.M., Feng, Y.M. and Shi, J.C. (2010) Hematoma Volume Measuring Software and Tada Formula: Comparison and Application. Hebei Medical Journal, 32, 2149-2150.

[7] Chen, G.L., Zhou, W., Ren, H.J. and Chen, W.Y. (2005) Clinical Significance of Cisterna Ambiens Changes in Acute Traumatic Brain Injury. Zhejiang Journal of Traumatic Surgery, 10, 14-15.

[8] Gao, L. (2013) Correctly Evaluating the Role of Intracranial Pressure Monitoring in the Treatment of Severe Traumatic Brain Injury. Chinese Journal of Traumatology, 29, 100-102.

[9] Farahvar, A., Gerber, L.M., Chiu, Y.L., Carney, N., Härtl, R. and Ghajar, J. (2012) Increased Mortality in Patients with Severe Traumatic Brain Injury Treated without Intracranial Pressure Monitoring. Journal of Neurosurgery, 117, 729734. http://dx.doi.org/10.3171/2012.7.JNS111816

[10] Talving, P., Karamanos, E., Teixeira, P.G., Skiada, D., Lam, L., Belzberg, H., Inaba, K. and Demetriades, D. (2013) Intracranial Pressure Monitoring in Severe Head Injury: Compliance with Brain Trauma Foundation Guidelines and Effect on Outcomes: A Prospective Study. Journal of Neurosurgery, 119, 1248-1254. http://dx.doi.org/10.3171/2013.7.JNS122255

[11] Chen, X.P. and Wang, J.P. (2013) Surgery Science. 8th Edition, People’s Medical Publishing House, Beijing.

[12] Jiang, P.X., Zhang, F., Shi, D.S., Bao, Q.C. and Wu, W.Y. (2013) Conservative Treatment of Acute Extradural Hematoma: Dynamic CT Scanning and Turnover Analysis. Zhejiang Journal of Traumatic Surgery, 18, 680-682. 


\section{Abbreviations}

GCS: Glasgow Coma Scale;

GOS: Glasgow Outcome Scale;

TBI: Traumatic Brain Injury. 